\title{
Challenges at the interface of control engineering and synthetic biology
}

\author{
Harrison Steel, Gabriele Lillacci, Mustafa Khammash, and Antonis Papachristodoulou
}

\begin{abstract}
Synthetic biology is a rapidly expanding field at the interface of the engineering and biological sciences which aims to apply rational design principles in biological contexts. Many natural processes utilise regulatory architectures that parallel those found in control and electrical engineering, which has motivated their implementation as part of synthetic biological constructs. Tools based upon control theoretical concepts can be used to design such systems, as well as to guide their experimental realisation. In this paper we provide examples of biological implementations of negative feedback systems, and discuss progress made toward realisation of other feedback and control architectures. We then outline major challenges posed by the design of such systems, particularly focusing on those which are specific to biological contexts and on which feedback control can have a significant impact. We explore future directions for work in the field, including new approaches for theoretical design of biological control systems, the utilisation of novel components for their implementation, and the potential for application of automation and machinelearning approaches to accelerate synthetic biological research.
\end{abstract}

\section{INTRODUCTION}

Synthetic biology is a rapidly expanding field at the interface of the engineering and biological sciences which aims to apply rational design principles in biological contexts. Synthetic biological systems can be designed to tackle real-world problems ranging from environmental cleanup to chemical synthesis [1], and from medical sensing to decision making and computation [2], [3]. Early breakthroughs in the field were achieved via the re-wiring of natural genetic components [4], [5], [6], followed by extensive trial-and-error experimentation to find appropriate parameter regimes for their operation. Though early efforts demonstrated the broad capabilities of synthetic biology, the field is far from reaching its potential [7]. Much must still be done to develop tools and design methodologies that account for the unreliable and often unpredictable behaviour of engineered biological systems [8], [9], which makes their application challenging in all but the most ideal conditions.

Control architectures similar to those traditionally used in control engineering [10], [11] have been found to govern a range of regulatory processes in natural systems. For example, autoregulatory architectures are ubiqutous

H. Steel and A. Papachristodoulou are with the Department of Engineering Science, University of Oxford, Oxford, OX1 3PJ, UK e-mail: harrison.steeldeng.ox.ac.uk, antonis@eng.ox.ac.uk. G. Lillacci and M. Khammash are with the Department of Biosystems Science and Engineering, ETH Zürich, Mattenstrasse 26, 4058, Basel, Switzerland, e-mail: gabriele.lillaccilbsse.ethz.ch, mustafa.khammashebsse.ethz.ch. H. Steel is supported in part by the General Sir John Monash foundation. A. Papachristodoulou is supported in part by EPSRC project EP/M002454/1. in both prokaryotes [12] and eukaryotes [13], which has motivated their synthetic implementation [12], [14], [15]. Other frequently studied natural control systems include the osmoregulatory mechanism in yeast [16] and the chemotaxis process in bacteria [17], both of which have been demonstrated to rely on architectures relevant to integral feedback control [18]. Synthetic control architectures that replicate the capabilities of their natural counterparts have been utilised for purposes including regulation of the unreliable nature of biological systems [19], and management of host organisms to boost their capabilities as bio-factories [20]. These synthetic control systems have been implemented using a diversity of biological components (including DNA, RNA, and Protein [15], [21], [22]), which can be combined to create architectures that operate on varying time-scales [23], [24] and species concentrations [25]. Though synthetic control systems have demonstrated useful properties in isolation their practical implementation as part of larger synthetic biological designs remains challenging [26], for reasons including a lack of systematic design tools or means for reliable interfacing between biological sub-systems [27].

Recent efforts to address these shortcomings have focused on standardisation of design processes and detailed characterisation of biological components [28]. This has included development of libraries of compatible biological parts that can be automatically assembled to provide computational functions [29], as well as design methodologies for the creation of genetic circuits that achieve common control goals such as adaptation and disturbance rejection [30], [31]. To further advance these designs it will be necessary to develop systems that approximate the fundamental components of traditional control systems, including integration, gain, and summation junctions [32], [33], which can be used to build Lead-Lag [27], PID [25], or more advanced controllers. Recent progress in this diretion has included demonstration of the first synthetic cellular integral feedback control system [34]. The selection of particular biological system architectures that replicate these functions and are in some sense optimal remains a major challenge, which is further complicated by the non-ideal interactions between components (for which load driver-type devices have recently been developed [35]). There is thus substantial scope for the development of theoretical design requirements and guidelines for biological control systems, which could then be used to inform their implementation. 


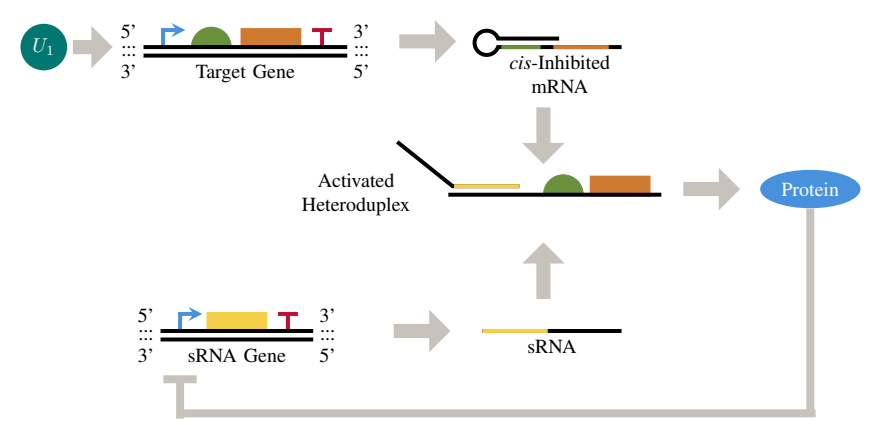

Fig. 1: An activating sRNA negative feedback architecture [36]. An inducer molecule $U_{1}$ controls the production rate of an mRNA which forms a hairpin secondary structure preventing access to its Ribosome Binding Site (RBS, green), inhibiting production of a protein-encoding gene (orange). In the presence of the corresponding sRNA this structure is altered due to base-pair binding between the sRNA and mRNA, allowing a protein to be produced. This protein down-regulates the production of sRNA (higher protein concentrations cause a decrease in sRNA production), forming a negative feedback loop.

In this tutorial paper we aim to provide an outline of the past progress and future challenges that lie at the intersection of control engineering and synthetic biology. We start in Section II by reviewing progress made toward the implementation of feedback architectures in biological contexts, before discussing other concepts from control engineering that have been adapted by synthetic biology in Section III. In Section IV the challenges posted by the design and implementation of such systems are described. In Section V potential directions for future work in the field are discussed, before the paper is concluded in Section VI.

\section{Feedback Control in Synthetic Biology}

The biological implementation of feedback control has been an ongoing goal of synthetic biology [37], [19], with early efforts focusing on its most elementary form, negative auto-regulation. These synthetic architectures demonstrated an ability to speed system response times, reduce noise, and linearise dose-responses [12], [14], [15], and have found practical implementation in metabolic engineering [38], [39]. Recent work has developed negative feedback systems using a diversity of genetic components [25], [40], including sRNA-based transcriptional regulation (Figure 1), integrase/excisionase based stochastic feedback (Figure 2), and transcription factor networks (Figure 3). Multicellular feedback control strategies which split modular control systems between communicating cellular hosts have also been demonstrated [41], which can improve system robustness and reduce the complexity of the synthetic system that must be sustained within each individual cell. Inter-cellular control strategies that utilise a variety of biological processes have been engineered, demonstrating their ability to control synchronised behaviours across a cell population [42], [43].

RNA based regulatory networks have been a recent focus of synthetic biological research due to their adaptability and ease of implementation [44], [45], [46], [47]. At a fundamental level they utilise the complementary basepairing between RNA strands to influence the rate at which the protein a gene encodes is read [48]. Such systems can be used to build relatively fast-acting feedback loops (Figure 1), and provide a means for realising summing junctions in biological contexts [36]. Systems based on the similar principle of DNA strand complementarity have been developed both theoretically and experimentally [49], [50], [51], and can be adapted for implementation of feedback control [22], [33]. Though the design of synthetic biological devices that utilise complementarity in such a way is conceptually straight-forward, challenges remain in the accurate a priori prediction of their behaviour, due to factors including their tendency to form complex secondary structures which impact their function [52].

Integrase proteins, which are used by viruses to integrate their genetic code with that of an invaded cell, can be re-engineered to flip a sequence of DNA between two specific recognition sites [54]. The recognition sites are (predictably) changed by this process, with the reverse flipping operation (to regain the DNA segment's original orientation) only occurring in the presence of a secondary protein (an excisionase). The presence (and concentration) of excisionase can thus be used to form a negative feedback loop by linking its expression to integrase-mediated DNA flipping (Figure 2). Such systems have been shown to reduce variability in the expression of genes under their control [53], which arise due to fluctuations in the rate at which genes are expressed between cells. Such variability between cells, which emerges due to both their internal processes and external environment [55], causes synthetic biological systems to behave unreliably, thus making its regulation desirable in many contexts [11].

Transcription factors have been recently used to implement antithetic integral feedback (AIF) [34], a way of realizing integral feedback that is particularly relevant to biomolecular networks [56]. In the antithetic topology, the regulation is carried out by two controller species, namely an actuator species (denoted $Z_{1}$ ) and a sensor species (denoted $Z_{2}$ ). A key feature of AIF is that the controller and the actuator annihilate each other, i.e. they abolish each other's biological activity upon reacting, for example by forming a high-affinity, biologically inert dimer.

The AIF circuit was implemented in Escherichia coli to control the expression of a protein of interest, which we denote $Y$. The promoter $\mathrm{P}_{Z_{1}}$ that responds to the actuator species drives a bicistronic construct consisting of the gene that codes for $Y$ and of a second gene coding for a fluorescent protein that is used as a proxy for $Y$. 


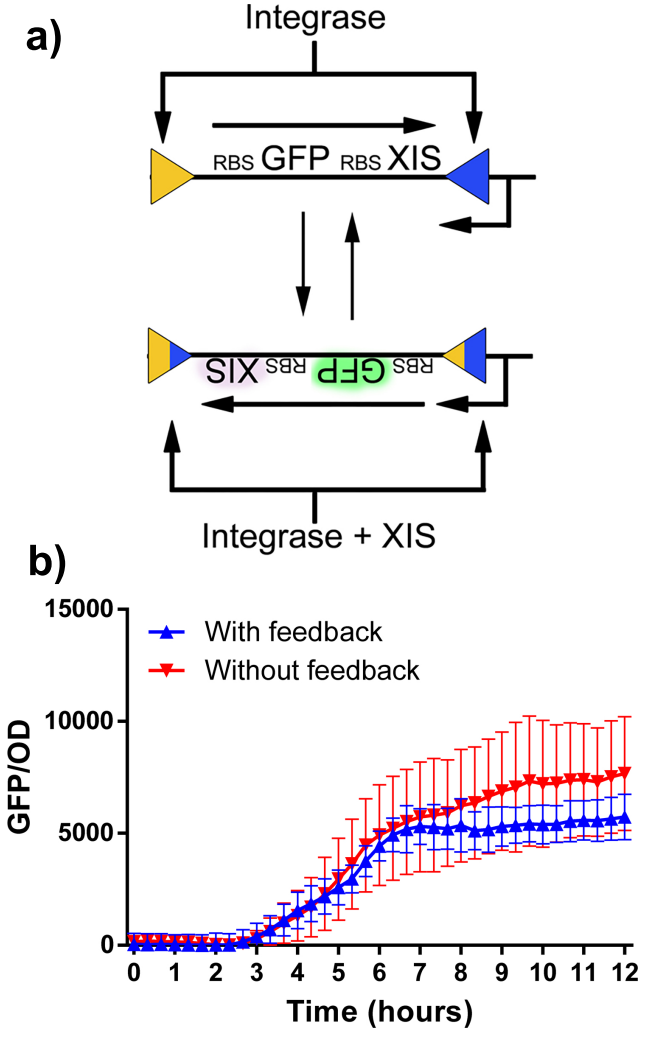

Fig. 2: An integrase-based negative feedback architecture, described in [53]. a) The system uses an integrase protein to flip a piece of DNA (top) framed by two recognition sites (yellow/blue triangles). Once this section of DNA is flipped (bottom) its contained reporter protein (Green Fluorescent Protein, GFP) and the corresponding excisionase (XIS) are produced. Together, integrase and the now-expressed excisionase can flip the DNA between the two new recognition sites back to the original DNA orientation. This system represents a negative feedback architecture that regulates the concentration of GFP/XIS, since the rate of reverse flipping is dependent on XIS concentration. b) Experimentally measured data demonstrating that the level of GFP fluorescence per cell is less variable (error bars denote the standard deviation in fluorescence for three bacterial colonies) in the feedback system described in a) when compared to a similar system that does not produce excisionase (meaning reverse DNA flipping does not occur).

The protein $Y$ also implements the feedback, as variations in the concentration of $Y$ are reflected in variations of activity of its cognate $\mathrm{P}_{Y}$ promoter, which in turn drives the gene for the sensor species. The response of $\mathrm{P}_{Y}$ to $Y$ can be adjusted with an inducer, referred to as $I_{2}$ : higher $I_{2}$ concentrations correspond to higher $\mathrm{P}_{Y}$ activity for a given $Y$ concentration. The expression of the actuator protein $Z_{1}$ is controlled by the inducible promoter $\mathrm{P}_{I_{1}}$, which can be regulated by another inducer $I_{1}$. Higher concentrations of $I_{1}$ result in higher $\mathrm{P}_{I_{1}}$ activity and increased production of the actuator species $Z_{1} . I_{1}$ and $I_{2}$ a)

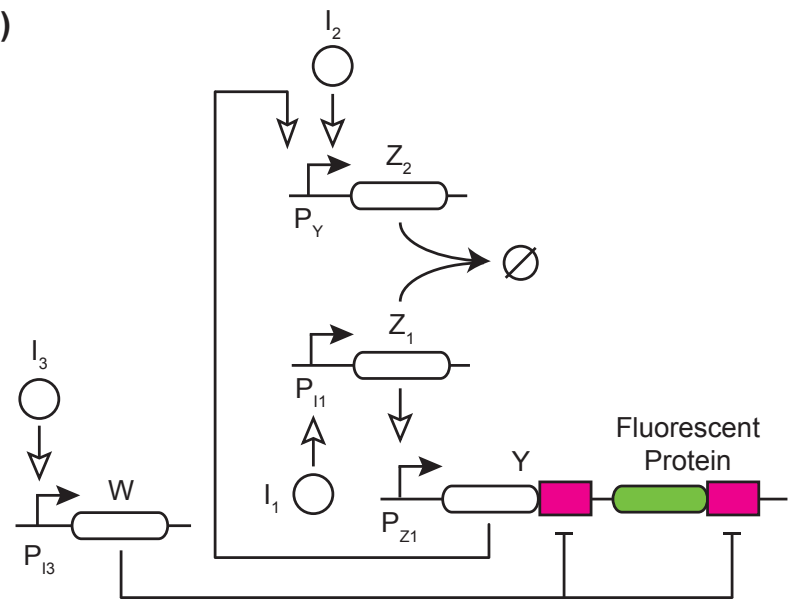

b)

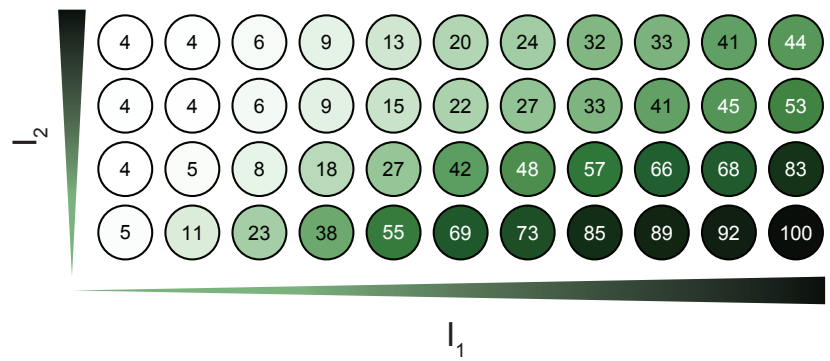

Fig. 3: Antithetic integral feedback in $E$. coli [34]. (a) Circuit schematic. Rounded rectangles indicate genes, purple rectangles show degradation tags, circles denote inducers. (b) Setpoint tunability. Steady-state fluorescence for cells containing the closed-loop AIF circuit grown in varying concentrations of inducers $I_{1}$ and $I_{2}$ (average of 2 replicates). The numbers indicate percentage of the maximal expression level.

can be used to tune the setpoint of the circuit, which is determined by the ratio of the production rates of $Z_{1}$ and $Z_{2}$ [56]. To test the disturbance rejection abilities of the circuit, a negative disturbance was implemented using an orthogonal protease denoted $W . W$ is driven by a third inducible promoter $\left(\mathrm{P}_{I_{3}}\right)$, controlled by the inducer $I_{3}$. W specifically recognized its cognate degradation tags, copies of which have been appended to C-termini of $Y$ and of the fluorescent protein. As a result, when $W$ is induced, the degradation rates of $Y$ and of the fluorescent protein are increased. The circuit is summarized in Fig. 3a. We refer to [34] for the specifics of the biological parts that were used.

The circuit was tested for its response to the two inducers $I_{1}$ and $I_{2}$. These were used to independently and systematically tune expression levels of $Z_{1}$ and $Z_{2}$, respectively, in all combinations. As expected, increasing $Z_{1}$ production corresponded to an increase in fluorescence, whereas increasing $Z_{2}$ production corresponded to a decrease (Fig. 3b). Furthermore, to show the effect of integral feedback, perfect or near-perfect adaptation was 
demonstrated in the presence of a persistent disturbance. The steady-state fluorescence was measured with and without induction of the protease $W$, in both the AIF closed-loop circuit and in an open-loop control lacking the feedback regulation. The closed-loop circuit showed no change in fluorescence, while the open-loop circuit showed a $50 \%$ decrease [34].

Like negative feedback, positive feedback architectures have been observed in a range of natural systems [57], and are now being included in synthetic biological designs [58]. In their most fundamental implementations, synthetic positive feedback loops have been used to amplify biological circuits' responses to inducers [59], and when nested with negative feedback circuits can provide robustness and tunability for circuits such as genetic oscillators [60]. Positive feedback is also of use in the creation of bi-stable switches, as it encourages fast and permanent transition between switch states [61]. Feed-forward loops, which can be created by combining positive and negative feedback [62], are well studied in natural processes [63], and provide topologies capable of perfect adaptation [64], [65] and fold-change detection [66], [67].

Beyond biological contexts, a variety of feedback controllers that interface with synthetic biological systems have been implemented in silico. Based on measurements of a cell colony's behaviour (for example, by measuring the fluorescence output of a synthetic circuit) such algorithms can estimate the control signal necessary to attain a desired outcome. Cells can then be manipulated to achieve this via a range of methods, such as input of inducer molecules [68], [69], or actuation using light-sensitive proteins [70]. These methods have been further developed in combination with automated experimental procedures that maintain cells in their exponential growth phase, providing hardware setups that can simultaneously regulate both cell growth and gene expression [71]. Setups which regulate cell growth in this manner can minimise many of the major sources of variability in synthetic biology, since cells can be maintained in their exponential growth phase in a nutrient-rich environment.

\section{Adaptation of Other Control Concepts}

In addition to the implementation of feedback and other regulatory architectures, recently progress has been made toward the adaptation of other core concepts from control engineering in synthetic biological contexts. For example, a molecular implementation of a least-meansquares estimator has been described [72], as have filtering architectures for state estimation, system identification, and noise cancellation [73]. Simple components from electrical circuits have also been under continual development, such as tunable amplification circuits [74].
The ability to make decisions that depend on multiple continuous or binary (on or off) inputs is another important control capability that has been recently developed in synthetic biological contexts [75]. Simple decision making circuits (such as AND gates) were initially designed and built using transcription factor networks [76], [77]. However, many such devices are functional only within a limited parameter range, and maintaining memory of a decision (e.g. one state of a bistable switch) can be challenging (though more complex designs that include positive feedback have improved upon these properties [61]). Recently, integrasebased decision circuits (which overcome some of these challenges) have received greater study [78]. Combinations of orthogonal integrases have been used to build many twoand three-input logic functions [29], which use transcription factors to process inputs, and integrases (expressed under their control) to re-arrange DNA cassettes. Integrase-based decision making circuits have the added benefit (compared to transcription factor-based decision making) of providing a reliable lasting record of past decisions; their outcome results in permanent changes to DNA that can be maintained for many $(>90)$ cell generations [79]. The permanence of integrase flipping also lends these systems to use in the design of circuits that record the order and relative timing of inputs [80], [81].

\section{Design AND IMPlEMENTATion OF BiologicAL CONTROL Systems}

A range of methods have been proposed to aid the design of synthetic biological networks that achieve specific control goals [82], [83]. The first challenge in this process is the definition of an appropriate network topology, which can be approached via traditional control-theoretical methods whilst considering some biological limitations on component interactions [31]. Constraints that must be satisfied for systems to achieve adaptation [26], disturbance rejection [30], or switch-like responses [84] have been explored, as have state-space approaches to finding design constraints of both linear [85] and non-linear [86] systems. Though these approaches can be used to find networks that are in some way minimal (for example, requiring the fewest reactions to realise), this does not guarantee that their implementation will be feasible, or that they will perform robustly. Particular parameter value regimes can be selected during the design phase such that these constraints are satisfied (though these must then be achieved in implementation), using methods that parallel those commonly used in control and electrical engineering. For example, mathematical treatments of biological oscillators (such as the repressilator [4]) can guide parameter selection to meet specific frequency or amplitude goals [43], [87]. Synthetic biological systems that combine processes operating on widely varying timescales provide a range of benefits [88], including reducing the challenge presented by realisation of specific network structures in order to guarantee certain control behaviours. For example, systems that use a layer of fast-acting control components to regulate 
a layer of slower processes shift design challenges away from definition of precise network structures, instead relying on selection and tuning of parameter values in the fast layer [23].

The tuning of feedback systems via adjustment of their individual parameters and components is an ongoing challenge in synthetic biology. As is known from control engineering, certain fundamental constraints limit achievable controller performance (for example, the waterbed effect has been observed in yeast [89]). Parameter tuning is often achieved by selecting Ribosome Binding Sites (RBSs) or promoters (which regulate translation and transcription respectively) of appropriate strengths [90], [91], though their behaviour can vary substantially depending on other cellular processes with which they interact [92]. Critical in this balance is cell burden, which is broadly used to describe the consumption of available cellular resources by synthetic circuits. Thus, if a synthetic (or natural) circuit is creating its components or products at a very high rate, the overall pool of free cellular resources (such as ribosomes and charged tRNAs) can be substantially reduced [93]. Cell burden may impact other cellular processes in a difficult-to-predict manner, potentially decreasing the rate at which certain proteins are produced [94] and reducing cell growth rate [95]. This can introduce secondary "virtual" network connections between elements of a synthetic circuit that are not designed to directly interact [96]. In many cases similar secondary effects and relationships can be responsible for context-dependence and unforeseen circuit behaviours [97], though recently a number of approaches have been developed via which they may be overcome. One such approach is the utilisation of orthogonal transcription and translation machinery [98], which because they are only used by the introduced synthetic systems, are not substantially disturbed by a cell's natural behavioural variations. However, such approaches do not address the more extreme host-circuit interactions, such as the potential for strong expression of synthetic genes to lead to retardation of their host's growth [99].

Another approach to overcoming the cellular burden of synthetic biological systems is to build circuits that function at low component abundances, or build upon native cellular machinery to reduce the number of additional genes that must be expressed [56]. Recent work has demonstrated that natural systems can be built upon and re-purposed in this way by as little as the addition of a single appropriately acting protein. For example, inclusion of a new interaction in a phosphorylation cycle can re-purpose a system with a robust step-response to provide a user-defined output level, or even near-perfect adaptation [100] (an example of such a system is described in Figure 4). This approach is doubly beneficial; the introduced cell burden is small (since only a single additional protein is expressed), and due to its simplicity, experimental realisation of such a control system can be relatively straightforward. When considering the

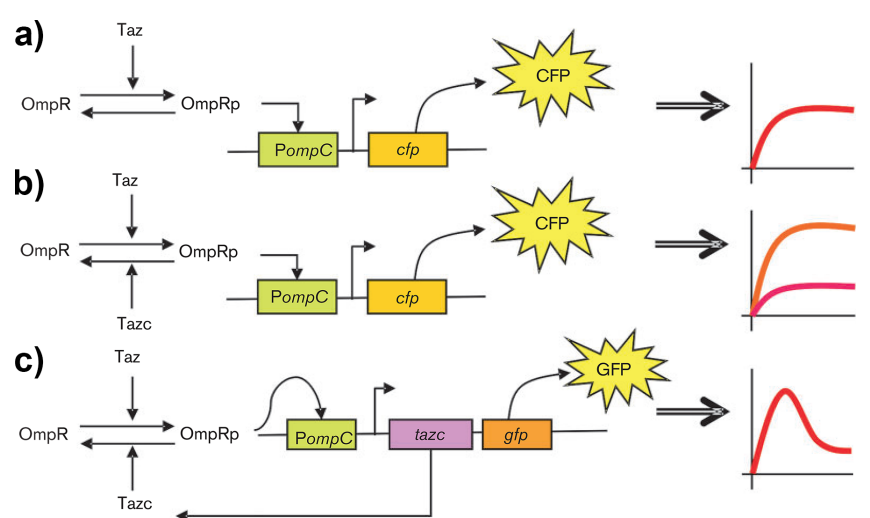

Fig. 4: Re-designing of the Taz-OmpR system [101] with addition of a single Phosphatase enzyme (Tazc). a) The original system, in which the protein Taz receives a stepinput via external stimulus, and encourages the reversible phosphorylation of OmpR to form OmpRp. OmpRp then binds to the ompC promoter (PompC), increasing the production rate of cfp (Cyan Fluorescent Protein). The system thus produces a step response fluorescence output. b) Addition of Tazc can shift the step response magnitude by de-phosphorylating OmpRp, with the output level of cfp dependent upon Tazc concentration. c) Placing the gene for Tazc downstream of PompC forms a feedback loop, where high OmpRp levels increase production of Tazc, thereby dephosphorylating OmpRp to form OmpR. By closing the loop the system can exhibit a near-adaptive response to a step input. Figure adapted with permission from [100].

burden imposed upon a cell by synthetic control systems, DNA-based systems are similarly attractive, since they do not rely on resource-intensive protein production, whilst being able to perform the information processing necessary for effective control [95].

In addition to burdening their host cells, synthetic biological systems can substantially influence the upand down-stream processes with which they interface in undesirable ways. For example, down-stream elements in a biological circuit can apply a "load" to connected upstream components by consuming (or temporarily sequestering) them [102]. This makes the utilisation of timing circuits (such as oscillators) to control other processes challenging, since any connections (i.e. secondary processes that interact with the components of the oscillatory circuit) will disrupt the timing circuit's own dynamics, affecting its period and amplitude, or potentially breaking it altogether. This effect is well studied in electrical engineering, where designs must include parts that "insulate" up-stream components from those with which they interface, and this has inspired study of similar situations in biological contexts [103]. To address this effect biological load-drivers (inspired by those in control and electrical engineering) have been developed, which use fast-acting phosphotransfer processes to provide an insulating buffer between slower components, almost 
eliminating previously potent loading effects [35]. Similar architectures can be used to implement amplifier-like circuits [104], which will likely find extensive use as synthetic biological circuits grow in size and diversity of constituent components.

For many biological processes, robustness (that is, a system's insensitivity to fluctuations in its components' performance and surrounding conditions) can be critical for reliable function [105]. Systems that are designed without consideration of how variation in their individual parameters will impact their performance are thus at the mercy of noisy biochemical processes, which can perturb or completely disrupt their desired behaviour [11]. Though for many synthetic constructs the regulation of noise is desirable for this reason [106], some natural systems benefit from its presence [107], [108], [109]. For example, variability between cells can provide an evolutionary advantage in changing environments [110]. Synthetic systems have been developed which similarly benefit from the stochasticity of gene expression [56], and it is often necessary to include noise in their mathematical treatment to reproduce fundamental aspects of their behaviour [111].

\section{Future DiRECTIONS}

\section{A. Re-considering design of synthetic biological systems}

Though much progress has been made toward the realization of control systems in synthetic biological contexts, a range of significant challenges must be overcome before they can find widespread practical implementation. Let us consider a biological circuit modelled by a system of nonlinear ordinary differential equations of the form:

$$
\dot{\mathbf{x}}=f(\mathbf{x}, \mathbf{u}, \mathbf{p})
$$

where $\mathbf{x}$ is a vector of system state variables (for example, protein or sRNA concentrations), $\dot{\mathbf{x}}$ is its time derivative, $\mathbf{u}$ is a vector of inputs to the system (for example, concentrations of inducer molecules), and $\mathbf{p}$ is a vector of parameters that enter the non-linear equations, $f$. The first challenge in designing a system of this form is defining the structure of $f$ such that the state variables $\mathbf{x}$ achieve a desirable steady state and dynamic behaviour. Future work will likely focus on approaching this problem in a fundamentally biological context, such that network topologies and implementations selected are biologically tractable, rather than by adapting or building upon methods previously developed in electrical and control engineering for which this consideration is not relevant. In some cases differential equation models such as this may be inappropriate, particularly when systems work at the single-molecule level, necessitating stochastic modelling.

The second major challenge is realising the desired structure of $f$ via the selection and tuning of reliable components and parts, thereby defining the parameters p. As discussed in Section IV this process must consider a range of secondary effects and interdependencies that arise in complex biological systems, as well as the fundamental limitations of their performance. Finally, with design frameworks and appropriate components in hand, synthetic biologists and control theorists will be able to implement control systems as part of larger synthetic constructs, going beyond previous work which has predominantly focused on the demonstration of control systems in isolation.

Due to the inherent noisiness of biological systems it is crucial that variability in the performance of their individual components is considered throughout the design process. As discussed in Section IV, many natural systems have highly robust architectures which allow them to function in variable environments and cellular states [11]. Though some synthetic designs have built upon and repurposed robust natural systems [100], the development of design methodologies that treat parameters as probability distributions, thereby allowing for substantial uncertainty in their values, has only recently begun [112]. Approaching design in such a way can aid in the tuning process of synthetic circuits, as adjustments can be made with the intention of shifting/shaping probability distributions (rather than just attempting to alter mean measured parameter values), maximising the potential that once implemented a system's behaviour will fall within acceptable limits. By carrying the uncertainty though the design and tuning process, such approaches may also reveal network topologies which are particularly robust, but may be currently overlooked due to their apparent complexity (e.g. number of components/reactions required for implementation).

As prior efforts in synthetic biology have revealed, many designs which may on paper appear uncomplicated can be difficult or even impossible to implement with current state-of-the-art biological components and experimental procedures. Selecting the most promising designs from a range of potential theoretical candidates thus requires extensive experimental expertise, as a combination of the practical behaviour of the components selected, as well as experimental procedures that might be followed to assemble them into a working system, must be considered. Past approaches have addressed this via modularity of parts and experimental procedures [29], but have been restricted to systems with limited sets of capabilities. There is therefore a great need for design procedures and theoretical treatments of biological systems which consider and quantify potential circuits in terms of "ease of implementation". Defining such a metric with any kind of reliability or standardisation would pose a significant challenge, particularly in terms of quantising the difficulty in using various combinations of parts and experimental procedures. Furthermore, it would require regular re-standardisation as the range of biological components and experimental procedures from which to choose grows. 


\section{B. New approaches to implementation}

Study of biological processes and systems across a variety of organisms has yielded a diverse (and growing) selection of genetic parts that can be utilised in synthetic biological designs [113]. However, at present synthetic constructs still frequently rely on transcriptional regulatory networks, which though relatively easy to design and implement, are slow-acting and resource-intensive [88]. In natural systems such networks are typically used for long-term cellular decision making, whilst faster control-related functionalities are enacted by protein-based interactions (such as the phosphorylation cycles discussed in Section IV) [88]. To produce similarly capable control networks in synthetic biological contexts it will likely be necessary to utilise approaches that rely on RNA-based mechanisms [114], or protein engineering, as has been done in some simple cases discussed previously [35]. Until now novel functionalities have been achieved by adapting or modifying naturally occurring proteins, however, recently much progress has been made in the rational design of functional proteins [113]. There is thus substantial room for development of biological circuits that utilise synthetic proteins, and future design philosophies will need to account for these rapidly expanding capabilities and their potential applications.

As the diversity of components with which synthetic biological systems can be built has grown, so too has the range of cellular chassis, or entirely cell-free systems, in which they can be implemented. At the most fundamental level are cell-free systems, which provide a minimal media containing pre-determined quantities of the cellular machinery necessary for protein expression (such as Ribosomes, nucleotides, and amino-acids) [115]. Such systems eliminate many background cellular processes whose interference with synthetic systems introduces noise, and they provide a relatively predictable breakdown of cellular chemicals and components. A range of attempts have been made to achieve expression environments of intermediary complexity (for example, SimCells [116]), which often involve removal of non-essential functions from a standard bacterial host [117]. By simplifying the environment in which synthetic biological systems are expressed each of these approaches allows more reliable prediction of their behaviour. Mathematical models and control theoretical treatment are thus more reliably applicable, and may be expanded with greater confidence to account for the interplay between synthetic systems and the surrounding cellular machinery.

\section{Automation and experimental standardisation}

As synthetic biologists have attempted to replicate experimental studies from the literature in their own laboratories it has become clear that due to the contextdependence of many synthetic circuits slight variations in experimental procedures and conditions can result in widely varying results. This has encouraged the standardisation and automation of experimental procedures via use of robotic platforms, which can reliably perform many of the tedious laboratory tasks that were previously completed manually by scientists and technicians. Moving a step further are companies such as Transcriptic [118], which offer remote automation of experimental procedures from start to finish: Scientists provide a DNA sequence or plasmid (for which synthesis methods have progressed rapidly in both capability and reliability in recent years [119]), and then computationally design a series of experiments and measurements. These can make use of standardised cell lines and reagents, and an ever growing selection of hardware, and are performed in the company's largely automated laboratories. A range of software tools have been developed to speed the design and implementation of such experiments, substantially reducing the knowledge-barrier to entry [120]. Automated approaches mean scientists do not need access to their own laboratory, thereby lowering the resource and experimental expertise requirements for participation in synthetic biological research.

As synthetic biological procedures and design tasks become more automated, there is an increasing need for machine learning approaches to be included in experimental workflows to maximise the amount that can be learnt from both successes and failures [121]. Past studies have utilised a diversity of machine learning tools for purposes including the identification of SRNA and ncRNA (non coding RNA) genes [122], [123], and companies with access to automated facilities employ such techniques to analyse the large data sets produced by biological experiments [124]. As the synthetic biological design cycle becomes increasingly automated and data-driven there will be a range of opportunities for application of novel machine learning techniques [125], which may even be implemented by biological systems [126].

\section{CONCLUSION}

The application of control theoretical thinking to synthetic biology has resulted in a range of landmark results over the past two decades. Many components that approximate those used in electrical and control engineering have been implemented in biological contexts, and have on occasion been utilised as part of larger circuits that tackle real-world problems. However, in the future control designs will likely need to move toward more advanced and robust feedback architectures, and do so by effectively using the growing diversity of biological components available for their implementation. Until now biological control systems have frequently been studied and implemented in isolation, often using inducer molecules as input and fluorescent reporters as output, which has not fully accounted for many of the context-dependent effects known to strongly impact synthetic systems. Moving forward, control architectures will thus need to be designed and integrated as part of larger synthetic biological constructs, which may then be 
used to address a diversity of problems in fields ranging from industrial chemical synthesis to healthcare.

\section{REFERENCES}

[1] M. Eisenstein, "Living Factories of the Future," Nature, vol. 531, no. 7594, pp. 401-403, 2016.

[2] J. J. Collins and A. S. Khalil, "Synthetic biology: applications come of age," Nature reviews Genetics, vol. 11, no. 5, pp. 367-379, 2010.

[3] W. C. Ruder, T. Lu, and J. J. Collins, "Synthetic biology moving into the clinic." Science (New York, N.Y.), vol. 333, no. 6047, pp. 1248-1252, 2011.

[4] M. B. Elowitz and S. Leibler, "A synthetic oscillatory network of transcriptional regulators," Nature, vol. 403, no. 6767, pp. 335-338, jan 2000.

[5] T. S. Gardner, C. R. Cantor, and J. J. Collins, "Construction of a genetic toggle switch in Escherichia coli," Nature, vol. 403, no. 6767, pp. 339-342, jan 2000.

[6] N. Nandagopal and M. B. Elowitz, "Synthetic Biology: Integrated Gene Circuits," Science, vol. 333, no. 6047, pp. 1244-1248, 2011.

[7] G. M. Church, M. B. Elowitz, C. D. Smolke, C. a. Voigt, and R. Weiss, "Realizing the potential of synthetic biology." Nature reviews. Molecular cell biology, vol. 15, no. 4, pp. 289-294, 2014.

[8] M. J. Dunlop, R. S. Cox, J. H. Levine, R. M. Murray, and M. B. Elowitz, "Regulatory activity revealed by dynamic correlations in gene expression noise." Nature genetics, vol. 40, no. 12, pp. $1493-$ $1498,2008$.

[9] A. A. K. Nielsen, T. H. Segall-Shapiro, and C. A. Voigt, "Advances in genetic circuit design: Novel biochemistries, deep part mining, and precision gene expression," Current Opinion in Chemical Biology, vol. 17, no. 6, pp. 878-892, 2013.

[10] D. Del Vecchio, A. J. Dy, and Y. Qian, "Control theory meets synthetic biology." Journal of the Royal Society, vol. 13, no. 120, pp. 3-43, 2016.

[11] M. Khammash, "An engineering viewpoint on biological robustness." BMC biology, vol. 14, no. 1, p. 22, 2016.

[12] N. Rosenfeld, M. B. Elowitz, and U. Alon, "Negative autoregulation speeds the response times of transcription networks," Journal of Molecular Biology, vol. 323, no. 5, pp. 785-793, 2002.

[13] T. I. Lee, N. J. Rinaldi, F. Robert, N. M. Hannett, C. T. Harbison, C. M. Thompson, I. Simon, J. Zeitunger, E. G. Jennings, H. L. Murray, D. Benjamin, T. L. Volkert, E. Fraenkel, D. K. Gifford, and R. A. Young, "Transcriptional Regulatory Networks in Saccharomyces cerevisiae," Science, vol. 298, no. October, pp. 799-804, 2002.

[14] A. Becskei and L. Serrano, "Engineering stability in gene networks by autoregulation." Nature, vol. 405, no. June, pp. 590-593, 2000.

[15] D. Nevozhay, R. M. Adams, K. F. Murphy, K. Josic, and G. Balázsi, "Negative autoregulation linearizes the dose-response and suppresses the heterogeneity of gene expression." PNAS, vol. 106, no. 13, pp. 5123-8, 2009.

[16] D. Muzzey, C. A. Gómez-Uribe, J. T. Mettetal, and A. van Oudenaarden, "A Systems-Level Analysis of Perfect Adaptation in Yeast Osmoregulation," Cell, vol. 138, no. 1, pp. 160-171, 2009.

[17] H. C. Berg and D. A. Brown, "Chemotaxis in Escherichia coli analysed by three-dimensional tracking." Nature, vol. 239, no. 5374, pp. 500-504, 1972.

[18] T. M. Yi, Y. Huang, M. I. Simon, and J. Doyle, "Robust perfect adaptation in bacterial chemotaxis through integral feedback control." PNAS, vol. 97, no. 9, pp. 4649-53, 2000.

[19] S. Chen, P. Harrigan, B. Heineike, J. Stewart-Ornstein, and H. ElSamad, "Building robust functionality in synthetic circuits using engineered feedback regulation," Current Opinion in Biotechnology, vol. 24, no. 4, pp. 790-796, 2013.

[20] P. P. Peralta-Yahya, F. Zhang, S. B. del Cardayre, and J. D. Keasling, "Microbial engineering for the production of advanced biofuels," Nature, vol. 488, no. 7411, pp. 320-328, 2012.

[21] J. Chappell, M. K. Takahashi, and J. B. Lucks, "Creating small transcription activating RNAs," Nature Chemical Biology, vol. 11, no. February, pp. 1-9, 2015.

[22] B. Yordanov, J. Kim, R. L. Petersen, A. Shudy, V. V. Kulkarni, and A. Phillips, "Computational design of nucleic acid feedback control circuits," ACS Synthetic Biology, vol. 3, no. 8, pp. 600-616, 2014.
[23] T. P. Prescott and A. Papachristodoulou, "Designing Conservation Relations in Layered Synthetic Biomolecular Networks," IEEE Transactions on Biomedical Circuits and Systems, vol. 9, no. 4, pp. 572-580, 2015.

[24] P. Rivera-Ortiz and D. Del Vecchio, "Integral action with time scale separation: A mechanism for modularity in biological systems," Proceedings of the IEEE Conference on Decision and Control, pp. 49-55, 2015.

[25] C. Briat, C. Zechner, and M. Khammash, "Design of a Synthetic Integral Feedback Circuit: Dynamic Analysis and DNA Implementation," ACS Synthetic Biology, vol. 5, no. 10, pp. 1108-1116, 2016

[26] J. Ang and D. R. McMillen, "Physical constraints on biological integral control design for homeostasis and sensory adaptation," Biophysical Journal, vol. 104, no. 2, pp. 505-515, 2013.

[27] A. W. K. Harris, J. A. Dolan, C. L. Kelly, J. Anderson, and A. Papachristodoulou, "Designing Genetic Feedback Controllers," IEEE Transactions on Biomedical Circuits and Systems, vol. 9, no. 4, pp. 475-484, 2015.

[28] B. Canton, A. Labno, and D. Endy, "Refinement and standardization of synthetic biological parts and devices," Nature Biotechnology, vol. 26, no. 7, pp. 787-793, 2008.

[29] A. A. K. Nielsen, B. S. Der, J. Shin, P. Vaidyanathan, V. Paralanov, E. A. Strychalski, D. Ross, D. Densmore, and C. A. Voigt, "Genetic circuit design automation." Science, vol. 352, no. 6281, p. aac7341, 2016.

[30] T. Drengstig, I. W. Jolma, X. Y. Ni, K. Thorsen, X. M. Xu, and P. Ruoff, "A basic set of homeostatic controller motifs," Biophysical Journal, vol. 103, no. 9, pp. 2000-2010, 2012.

[31] H. Steel and A. Papachristodoulou, "Constraints for Biological Systems that Achieve Adaptation and Disturbance Rejection," Submitted paper, 2017.

[32] R. Daniel, J. R. Rubens, R. Sarpeshkar, and T. K. Lu, "Synthetic analog computation in living cells," Nature, vol. 497, no. 7451, pp. 619-623, 2013.

[33] K. Oishi and E. Klavins, "Biomolecular implementation of linear I/O systems," IET Systems Biology, vol. 5, no. 4, pp. 252-260, 2011.

[34] G. Lillacci, S. Aoki, D. Schweingruber, and M. Khammash, "A synthetic integral feedback controller for robust tunable regulation in bacteria," bioRxiv, pp. 1-7, 2017.

[35] D. Mishra, P. M. Rivera, A. Lin, D. Del Vecchio, and R. Weiss, "A load driver device for engineering modularity in biological networks," Nature Biotechnology, vol. 32, no. 12, pp. 1268-1275, 2014.

[36] H. Steel, A. W. K. Harris, E. J. Hancock, C. L. Kelly, and A. Papachristodoulou, "Frequency domain analysis of small non-coding RNAs shows summing junction-like behaviour," Proceedings of the 56th IEEE Conference on Decision and Control., 2017.

[37] R. M. Murray and D. Del Vecchio, "Biomolecular Feedback Systems," Bernoulli, 2010.

[38] F. Zhang, J. M. Carothers, and J. D. Keasling, "Design of a dynamic sensor-regulator system for production of chemicals and fuels derived from fatty acids." Nature biotechnology, vol. 30, no. 4, pp. 354-9, 2012.

[39] F. He, E. Murabito, and H. V. Westerhoff, "Synthetic biology and regulatory networks: where metabolic systems biology meets control engineering," Journal of the Royal Society, Interface, vol. 13, p. 20151046, 2016.

[40] J.-C. Lin and D. Thirumalai, "Gene regulation by riboswitches with and without negative feedback loop." Biophysical Journal, vol. 103, no. 11, pp. 2320-30, 2012.

[41] G. Fiore, A. Matyjaszkiewicz, F. Annunziata, C. Grierson, N. J. Savery, L. Marucci, and M. Di Bernardo, "In-Silico Analysis and Implementation of a Multicellular Feedback Control Strategy in a Synthetic Bacterial Consortium," ACS Synthetic Biology, vol. 6, no. 3, pp. 507-517, 2017.

[42] M. O. Din, T. Danino, A. Prindle, M. Skalak, J. Selimkhanov, K. Allen, E. Julio, E. Atolia, L. S. Tsimring, S. N. Bhatia, and J. Hasty, "Synchronized cycles of bacterial lysis for in vivo delivery." Nature, vol. 536, no. 7614, pp. 81-85, 2016.

[43] L. Potvin-Trottier, N. D. Lord, G. Vinnicombe, and J. Paulsson, "Synchronous long-term oscillations in a synthetic gene circuit," Nature, vol. 538, no. 7626, pp. 514-517, 2016.

[44] S. Gottesman and G. Storz, "Bacterial small RNA regulators: Versatile roles and rapidly evolving variations," Cold Spring Harbor Perspectives in Biology, vol. 3, no. 12, pp. 1-16, 2011. 
[45] D. Na, S. M. Yoo, H. Chung, H. Park, J. H. Park, and S. Y. Lee, "Metabolic engineering of Escherichia coli using synthetic small regulatory RNAs." Nature biotechnology, vol. 31, no. 2, pp. 1704, 2013.

[46] E. Franco, G. Giordano, P. O. Forsberg, and R. M. Murray, "Negative autoregulation matches production and demand in synthetic transcriptional networks," ACS Synthetic Biology, vol. 3, no. 8, pp. 589-599, 2014.

[47] A. N. Leistra, P. Amador, A. Buvanendiran, A. Moon-Walker, and L. M. Contreras, "Rational Modular RNA Engineering Based on In Vivo Profiling of Structural Accessibility," ACS Synthetic Biology, p. acssynbio.7b00185, 2017.

[48] A. A. Green, P. A. Silver, J. J. Collins, and P. Yin, "Toehold switches: De-novo-designed regulators of gene expression," Cell, vol. 159, no. 4, pp. 925-939, 2014.

[49] D. Soloveichik, G. Seelig, and E. Winfree, "DNA as a universal substrate for chemical kinetics," Lecture Notes in Computer Science (including subseries Lecture Notes in Artificial Intelligence and Lecture Notes in Bioinformatics), vol. 5347 LNCS, no. 12, pp. 57-69, 2009.

[50] D. Y. Zhang and G. Seelig, "Dynamic DNA nanotechnology using strand-displacement reactions." Nature chemistry, vol. 3, no. 2, pp. 103-113, 2011.

[51] T. Song, S. Garg, R. Mokhtar, H. Bui, and J. Reif, "Analog Computation by DNA Strand Displacement Circuits," ACS Synthetic Biology, p. acssynbio.6b00144, 2016.

[52] D. Y. Zhang, "Towards Domain-Based Sequence Design for DNA Strand Displacement Reactions," in Internatonal Workshop on DNABased Computers. Springer Berlin Heidelberg, 2010, pp. 162-175.

[53] T. Folliard, H. Steel, T. P. Prescott, G. Wadhams, L. J. Rothschild, and A. Papachristodoulou, "A synthetic recombinase-based feedback loop results in robust expression," ACS Synthetic Biology, 2017.

[54] N. D. Grindley, K. L. Whiteson, and P. A. Rice, "Mechanisms of SiteSpecific Recombination,” Аnnu. Rev. Biochem., vol. 75, pp. 567-605, 2006.

[55] P. S. Swain, M. B. Elowitz, and E. D. Siggia, "Intrinsic and extrinsic contributions to stochasticity in gene expression." Proceedings of the National Academy of Sciences of the United States of America, vol. 99 , no. 20, pp. $12795-800,2002$.

[56] C. Briat, A. Gupta, and M. Khammash, "Antithetic Integral Feedback Ensures Robust Perfect Adaptation in Noisy Biomolecular Networks," Cell Systems, vol. 2, no. 1, pp. 15-26, 2016.

[57] J. L. Radzikowski, S. Vedelaar, D. Siegel, Á. D. Ortega, A. Schmidt, and M. Heinemann, "Bacterial persistence is an active $\mathrm{r} S$ stress response to metabolic flux limitation," Molecular systems biology, vol. 12, no. 882, pp. 1-18, 2016.

[58] A. W. K. Harris, H. Steel, C. L. Kelly, and A. Papachristodoulou, "The Autorepressor: a Case Study of the Importance of Model Selection," Proceedings of the 56th IEEE Conference on Decision and Control., 2017

[59] G. J. Nistala, K. Wu, C. V. Rao, and K. D. Bhalerao, "A modular positive feedback-based gene amplifier." Journal of biological engineering, vol. 4, p. 4, 2010.

[60] J. Stricker, S. Cookson, M. R. Bennett, W. H. Mather, L. S. Tsimring, and J. Hasty, "A fast, robust and tunable synthetic gene oscillator." Nature, vol. 456, no. 7221, pp. 516-519, 2008.

[61] T. Shopera, W. R. Henson, A. Ng, Y. J. Lee, K. Ng, and T. S. Moon, "Robust, tunable genetic memory from protein sequestration combined with positive feedback," Nucleic Acids Research, vol. 43, no. 18, pp. 9086-9094, 2015.

[62] S. Mangan and U. Alon, "Structure and function of the feedforward loop network motif." Proceedings of the National Academy of Sciences of the United States of America, vol. 100, no. 21, pp. 11 980-11 985, 2003.

[63] K. Papenfort, E. Espinosa, J. Casadesús, and J. Vogel, "Small RNAbased feedforward loop with AND-gate logic regulates extrachromosomal DNA transfer in Salmonella." Proceedings of the National Academy of Sciences of the United States of America, vol. 112, no. 34, pp. E4772-81, 2015.

[64] W. Ma, A. Trusina, H. El-Samad, W. A. Lim, and C. Tang, "Defining Network Topologies that Can Achieve Biochemical Adaptation," Cell, vol. 138, no. 4, pp. 760-773, 2009.

[65] J. Kim, I. Khetarpal, S. Sen, and R. M. Murray, "Synthetic circuit for exact adaptation and fold-change detection," Nucleic Acids Research, vol. 42, no. 9, pp. 6078-6089, 2014.
[66] L. Goentoro, O. Shoval, M. W. Kirschner, and U. Alon, "The Incoherent Feedforward Loop Can Provide Fold-Change Detection in Gene Regulation," Molecular Cell, vol. 36, no. 5, pp. 894-899, 2009.

[67] K. Kamino, Y. Kondo, A. Nakajima, M. Honda-Kitahara, K. Kaneko, and S. Sawai, "Fold-change detection and scale invariance of cellcell signaling in social amoeba," Proceedings of the National Academy of Sciences, pp. 24-26, 2017.

[68] F. Menolascina, G. Fiore, E. Orabona, L. De Stefano, M. Ferry, J. Hasty, M. di Bernardo, and D. di Bernardo, "In-Vivo Real-Time Control of Protein Expression from Endogenous and Synthetic Gene Networks," PLoS Computational Biology, vol. 10, no. 5, p. e1003625, may 2014

[69] V. Kulkarni, E. Kharisov, N. Hovakimyan, and J. Kim, "Load capacity improvements in nucleic acid based systems using partially open feedback control," ACS Synthetic Biology, vol. 3, no. 8, pp. 617626, 2014.

[70] A. Milias-Argeitis, S. Summers, J. Stewart-Ornstein, I. Zuleta, D. Pincus, H. El-Samad, M. Khammash, and J. Lygeros, "In silico feedback for in vivo regulation of a gene expression circuit." Nature biotechnology, vol. 29, no. 12, pp. 1114-6, 2011.

[71] A. Milias-Argeitis, M. Rullan, S. K. Aoki, P. Buchmann, and M. Khammash, "Automated optogenetic feedback control for precise and robust regulation of gene expression and cell growth," Nature Communications, vol. 7, no. May, p. 12546, 2016.

[72] C. Zechner and M. Khammash, "A Molecular Implementation of the Least Mean Squares Estimator," Proc. of IEEE Conference on Decision and Control, no. Cdc, 2016.

[73] C. Zechner, G. Seelig, M. Rullan, and M. Khammash, "Molecular circuits for dynamic noise filtering," Proceedings of the National Academy of Sciences, vol. 113, no. 17, pp. 4729-4734, 2016.

[74] B. Wang, M. Barahona, and M. Buck, "Engineering modular and tunable genetic amplifiers for scaling transcriptional signals in cascaded gene networks," Nucleic Acids Research, vol. 42, no. 14, pp. 9484-9492, 2014.

[75] O. Purcell and T. K. Lu, "Synthetic analog and digital circuits for cellular computation and memory," Current Opinion in Biotechnology, vol. 29, pp. 146-155, 2014.

[76] N. E. Buchler, U. Gerland, and T. Hwa, "On schemes of combinatorial transcription logic." Proceedings of the National Academy of Sciences of the United States of America, vol. 100, no. 9, pp. 513641, 2003.

[77] B. Wang, R. I. Kitney, N. Joly, and M. Buck, "Engineering modular and orthogonal genetic logic gates for robust digital-like synthetic biology," Nature Communications, vol. 2, no. 508, 2011.

[78] J. Bonnet, P. Yin, M. E. Ortiz, P. Subsoontorn, and D. Endy, "Amplifying genetic logic gates." Science (New York, N.Y.), vol. 340 , no. 6132, pp. 599-603, 2013.

[79] P. Siuti, J. Yazbek, and T. K. Lu, "Synthetic circuits integrating logic and memory in living cells." Nature biotechnology, vol. 31, no. 5, pp. 448-52, 2013.

[80] V. Hsiao, Y. Hori, P. W. K. Rothemund, and R. M. Murray, "A population-based temporal logic gate for timing and recording chemical events," Molecular systems biology, vol. 557, pp. 1-17, 2016.

[81] N. Roquet, A. P. Soleimany, A. C. Ferris, S. Aaronson, and T. K. Lu, "Synthetic recombinasebased state machines in living cells," Science, 2016.

[82] E. Appleton, C. Madsen, N. Roehner, and D. Densmore, "Design Automation in Synthetic Biology," Cold Spring Harbor Perspectives in Biology, 2017.

[83] I. Otero-Muras and J. R. Banga, "Exploring Design Principles of Gene Regulatory Networks via Pareto Optimality," IFACPapersOnLine, vol. 49, no. 7, pp. 809-814, 2016.

[84] N. A. Shah and C. A. Sarkar, "Robust network topologies for generating switch-like cellular responses," PLoS Computational Biology, vol. 7 , no. 6,2011 .

[85] S. U. Wei, "Topologies Needed for Perfect Adaptation," Proceedings of the 30th Chinese Control Conference, pp. 5714-5717, 2011.

[86] - "Perfect adaptation of general nonlinear systems," Journal of Systems Science and Complexity, vol. 29, no. 1, pp. 61-73, 2016.

[87] E. Lambert, E. J. Hancock, and A. Papachristodoulou, "Engineering a Genetic Oscillator Using Delayed Feedback," in Delay Systems, Advances in Delays and Dynamics Volume 1, 2014, pp. 389-402.

[88] R. M. Gordley, R. E. Williams, C. J. Bashor, J. E. Toettcher, S. Yan, and W. A. Lim, "Engineering dynamical control of cell 
fate switching using synthetic phospho-regulons." Proceedings of the National Academy of Sciences of the United States of America, vol. 113, no. 47, pp. 13528-13533, 2016.

[89] A. Mitchell, P. Wei, and W. A. Lim, "Oscillatory stress stimulation uncovers an Achilles heel of the yeast MAPK signaling network," Science, vol. 350, no. 6266, pp. 1379-1383, 2015.

[90] V. K. Mutalik, J. C. Guimaraes, G. Cambray, C. Lam, M. J. Christoffersen, Q.-A. Mai, A. B. Tran, M. Paull, J. D. Keasling, A. P. Arkin, and D. Endy, "Precise and reliable gene expression via standard transcription and translation initiation elements." Nature methods, vol. 10, no. 4, pp. 354-60, 2013.

[91] J. A. J. Arpino, E. J. Hancock, J. Anderson, M. Barahona, G. B. V. Stan, A. Papachristodoulou, and K. Polizzi, "Tuning the dials of synthetic biology," Microbiology (United Kingdom), vol. 159, no. PART7, pp. 1236-1253, 2013.

[92] D. Del Vecchio, "Modularity, context-dependence, and insulation in engineered biological circuits," Trends in Biotechnology, vol. 33, no. 2, pp. 111-119, 2015.

[93] J. L. Snoep, L. P. Yomano, H. V. Westerhoff, and L. O. Ingram, "Protein burden in Zymomonas mobilis: Negative flux and growth control due to overproduction of glycolytic enzymes," Microbiology, vol. 141, no. 9, pp. 2329-2337, 1995.

[94] A. Gyorgy, J. I. Jiménez, J. Yazbek, H. H. Huang, H. Chung, R. Weiss, and D. Del Vecchio, "Isocost Lines Describe the Cellular Economy of Genetic Circuits," Biophysical Journal, vol. 109, no. 3, pp. 639-646, 2015

[95] N. Roquet and T. K. Lu, "Digital and analog gene circuits for biotechnology," pp. 597-608, 2014.

[96] Y. Qian and D. Del Vecchio, "Mitigation of ribosome competition through distributed sRNA feedback," Proc. of IEEE Conference on Decision and Control, pp. 1-30, 2016.

[97] S. Cardinale and A. P. Arkin, "Contextualizing context for synthetic biology - identifying causes of failure of synthetic biological systems," Biotechnology Journal, vol. 7, no. 7, pp. 856-866, 2012.

[98] W. An and J. W. Chin, "Synthesis of orthogonal transcriptiontranslation networks." Proceedings of the National Academy of Sciences of the United States of America, vol. 106, no. 21, pp. 847782, 2009.

[99] C. Tan, P. Marguet, and L. You, "Emergent bistability by a growthmodulating positive feedback circuit," Nature Chemical Biology, vol. 5, no. 11, pp. 842-848, 2009.

[100] Y. C. Chang, J. P. Armitage, A. Papachristodoulou, and G. H. Wadhams, "A single phosphatase can convert a robust step response into a graded, tunable or adaptive response," Microbiology (United Kingdom), vol. 159, no. PART7, pp. 1276-1285, 2013.

[101] S. A. Forst and D. L. Roberts, "Signal transduction by the EnvZOmpR phosphotransfer system in bacteria," Research in Microbiology, vol. 145, no. 5-6, pp. 363-373, 1994.

[102] A. Gyorgy and D. Del Vecchio, "Modular Composition of Gene Transcription Networks," PLoS Computational Biology, vol. 10, no. 3, 2014.

[103] D. Del Vecchio, A. J. Ninfa, and E. D. Sontag, "Modular cell biology: retroactivity and insulation," Molecular systems biology, vol. 4, no. $161,2008$.

[104] K. S. Nilgiriwala, J. Jimenez, P. M. Rivera, and D. Del Vecchio, "Synthetic Tunable Amplifying Buff er Circuit in E. coli," ACS Synthetic Biology, vol. 4, pp. 577-584, 2015.

[105] H. Kitano, "Biological robustness," Nature Reviews Genetics, vol. 5, no. November, pp. 826-837, 2004.

[106] Y. Dublanche, K. Michalodimitrakis, N. Kummerer, M. Foglierini, and L. Serrano, "Noise in transcription negative feedback loops: simulation and experimental analysis," Mol Syst Biol, vol. 2, p. 41, 2006.

[107] M. Elowitz, A. Levine, E. Siggia, and P. Swain, "Stochastic gene expression in a single cell," Science, vol. 297, no. 5584, pp. 1183-6, 2002.

[108] L. T. Macneil and A. J. M. Walhout, "Gene regulatory networks and the role of robustness and stochasticity in the control of gene expression," Genome Research, pp. 645-657, 2011.

[109] G. Rodrigo and J. F. Poyatos, "Genetic Redundancies Enhance Information Transfer in Noisy Regulatory Circuits," PLoS Computational Biology, vol. 12, no. 10, pp. 1-20, 2016.

[110] G. Balazsi, A. Van Oudenaarden, and J. J. Collins, "Cellular decision making and biological noise: From microbes to mammals," Cell, vol. 144, no. 6, pp. 910-925, 2011.
[111] T. Tian and K. Burrage, "Stochastic models for regulatory networks of the genetic toggle switch." Proceedings of the National Academy of Sciences of the United States of America, vol. 103, no. 22, pp. 8372-8377, 2006.

[112] J. Ruess, H. Koeppl, and C. Zechner, "Sensitivity estimation for stochastic models of biochemical reaction networks in the presence of extrinsic variability," The Journal of Chemical Physics, vol. 146, no. 12, p. 124122, 2017.

[113] R. W. Bradley, M. Buck, and B. Wang, "Tools and Principles for Microbial Gene Circuit Engineering," Journal of Molecular Biology, vol. 428, no. 5, pp. 862-888, 2016.

[114] T. Folliard, B. Mertins, H. Steel, T. Prescott, T. Newport, C. Jones, G. Wadhams, T. Bayer, J. Armitage, A. Papachristodoulou, and L. Rothschild, "Ribo-attenuators: novel elements for reliable and modular riboswitch engineering," Scientific Reports, 2017.

[115] C. E. Hodgman and M. C. Jewett, "Cell-free synthetic biology Thinking outside the cell," Metabolic Engineering, vol. 14, no. 3, pp. 261-269, 2012.

[116] C. P. N. Rampley, P. A. Davison, P. Qian, G. M. Preston, C. N Hunter, I. P. Thompson, L. J. Wu, and W. E. Huang, "Development of SimCells as a novel chassis for functional biosensors," Scientific Reports, vol. 7, no. 1, p. 7261, 2017.

[117] C. A. Hutchison III, R.-y. Chuang, V. N. Noskov, N. Assad-garcia, T. J. Deerinck, M. H. Ellisman, J. Gill, K. Kannan, B. J. Karas, L. Ma, J. F. Pelletier, Z.-q. Qi, R. A. Richter, E. A. Strychalski, L. Sun, Y. Suzuki, B. Tsvetanova, K. S. Wise, H. O. Smith, J. I. Glass, C. Merryman, D. G. Gibson, and J. C. Venter, "Design and synthesis of a minimal bacterial genome," Science, vol. 351, no. 6280, 2016.

[118] Transcriptic, "Your Lab In The Cloud," 2017. [Online]. Available: https://www.transcriptic.com/

[119] R. A. Hughes and A. D. Ellington, "Synthetic DNA Synthesis and Assembly : Putting the Synthetic in Synthetic Biology," Cold Spring Harbor Perspectives in Biology, vol. 9, 2017.

[120] M. Bates, A. J. Berliner, P. R. Jaschke, and E. S. Groban, "Wet Lab Accelerator: A Web-Based Application Democratizing Laboratory Automation for Synthetic Biology," ACS Synthetic Biology, 2017.

[121] J. Nielsen and J. D. Keasling, "Engineering Cellular Metabolism," Cell, vol. 164, no. 6, pp. 1185-1197, 2016.

[122] R. K. Barman, A. Mukhopadhyay, and S. Das, "An improved method for identification of small non-coding RNAs in bacteria using support vector machine," Scientific reports, vol. 7, 2017.

[123] Q. Abbas, S. M. Raza, A. A. Biyabani, and M. A. Jaffar, "A Review of Computational Methods for Finding Non-Coding RNA Genes," Genes, vol. 7, no. 113, 2016.

[124] M. Bomgardner and E. N. W. Coast, "Start-ups with robots seek to scale up synthetic biology," vol. Chemical \&, pp. 18-22, 2016.

[125] J. Mellor, I. Grigoras, P. Carbonell, and J.-L. Faulon, "Semisupervised Gaussian Process for Automated Enzyme Search," ACS Synthetic Biology, p. acssynbio.5b00294, 2016.

[126] M. R. Lakin and D. Stefanovic, "Supervised Learning in Adaptive DNA Strand Displacement Networks," ACS Synthetic Biology, vol. 5, pp. 885-897, 2016. 